\title{
Invasive and the largest pancreatic tumour of Rare Intraabdominal Desmoid-type fibromatosis with curative resection: case report
}

\author{
Hanan Alghamdi ${ }^{1}$ \\ ${ }^{1}$ Imam Abdulrahman Bin Faisal University College of Medicine
}

April 25, 2021

\begin{abstract}
fibromatoses (Desmoid-type) tumour is rare benign fibrous tumours account approximately for $0.03 \%$ of all neoplasms. The Pancreas origin of this tumour is even more rarely reported subset with around $5 \%$ only. We aim to report the success in cure a rare and the largest pancreatic desmoid-type
\end{abstract}

\section{Case report:}

Invasive and the largest pancreatic tumour of Rare Intraabdominal Desmoid-type fibromatosis with curative resection: case report

\section{Hanan M Alghamdi}

King Fahad Hospital of the University, Imam Abdulrahman Bin Faisal University College of Medicine, Department of Surgery,

Running title: Intraabdominal fibromatosis

Keywords : Desmoid type fibromatosis, Desmoid tumour, fibromatosis, Pancreatic desmoid tumour, Radical pancreas resection, S100 protein, $\beta$-catenin, case report.

Correspondence to:

\section{Dr. Hanan M Alghamdi, MD}

Assistant Professor, Hepatobiliary \& Multiorgan Transplant \& Laparoscopic Surgeon

King Fahad Hospital of the University, college of medicine, Department of Surgery University of Imam Abdurahman Bin Faisal.

PO Box 40020, Al Khobar 31952, Saudi Arabia

Mobile: +966-(0)502828333

hmalghamdi@iau.edu.sa

hananghamdi@yahoo.com

\section{Key clinical message}

Radical-margin-free surgical resection for fibromatoses (Desmoid-type) tumour remains the mainstay management approach while chemotherapy and/or radiotherapy are not effective and no standardized protocol of consensuses and considered the last resort.

\section{Abstract}


Background:fibromatoses (Desmoid-type) tumour is rare benign fibrous tumours account approximately for $0.03 \%$ of all neoplasms. The Pancreas origin of this tumour is even more rarely reported subset with around 5\% only. Establishing the diagnosis is fundamentally based on characteristic pathological and immunohistochemical studies. Surgical resection of the tumour is the mainstay of intervention. We aim to report the success in cure a rare and the largest pancreatic desmoid-type intraabdominal fibromatosis.

\section{Case Presentation:}

A case of a 42 years old African-origin male who were medically free apart from recurrent left upper abdominal pain for more than 10 years, and no significant past surgeries, trauma, or cancer history in his family. By physical examination, the patient has a non-tender large abdominal mass at left hypochondrial area extending down to the pelvis below the umbilicus with rigid and smooth surface. The computed tomography scan showed large heterogenous mass which appears to be of pancreatic origin (the body and tail cannot be visualized due to this mass measuring about $23 \mathrm{~cm}$ by $15 \mathrm{~cm}$ by $11 \mathrm{~cm}$. The patient underwent radical en-mass resection with extended left pancreatectomy, segmental transverse colectomy, splenectomy, adrenalectomy, and subsequent colo-colic anastomosis. Histopathology and immunohistochemistry confirmed the diagnosis of pancreas fibromatosis desmoid- type. The follow up for the patient completed up to 5 years and no recurrence clinically or by ultrasound demonstrated.

Conclousion : our case is a rare and uniquely the largest pancreatic desmoid-type fibromatosis to be reported in the literature with curative resection despite being locally invasive to adjacent organ.

\section{Introduction:}

Desmoid tumour (DT) also called desmoid-type fibromatosis and aggressive fibromatosis, consider to be benign neoplasm in nature but locally invasive giving it the aggressive behaviour and fatal extent in advanced stages but without potential predilection for distant metastasis. The origin of DT is through proliferation from well differentiated mesenchymal fibroblasts. Types of DT (desmoids tumour) fibromatosis either abdominal wall, Extra-abdominal fibromatosis: mostly in the chest wall muscle, shoulder, thigh and back or Intra-abdominal fibromatosis originating from the mesentery or locating in the retroperitoneal and pelvic region or associated specifically with Gardner's syndrome. The consistency of DT mass varies between cystic, solid, or mixed solid and cystic form. The majority of DT present as extra-abdominal mass (in the muscle of the abdominal wall) and less commonly as intra-abdominally, occasionally can raise from any fibrous connective tissues in any location $[1,2]$.

Several possible risk factors or associated conditions reported in the literature may attribute to the development of DT include the history of DT in the family, exposure to irradiation, trauma, oral contraceptives bills use or pregnancy, some genetic mutation, and in familial adenomatosis polyposis (FAP) in 10-20\% of cases or Gardner syndrome, nevertheless, there is no definitive aetiology of DT [1-7]. Desmoid-type fibromatosis tumours diagnosis is primarily based on pathological and immunohistochemical studies features. Surgical resection of the tumour is the primary option.

The presented case is a rare DT occupying the whole distal part of the pancreas up to the neck of the pancreas, furthermore, it is considered to be the largest pancreatic mass to be ever reported in the literature and the first case of a pancreatic DT in Saudi Arabia, diagnosed in October 2016, at the.

\section{Case presentation}

A 42-year-old African origin male patient who had no comorbidities or previous surgeries or trauma history and no family history of cancer, Familial adenomatous polyposis syndrome, Gardner syndrome or cancer. His childhood was unremarkable, fully vaccinated, and never been hospitalized. Presented with complaints of recurrent left upper abdominal pain for more than 10 years and transferred to our hospital for suspicious large splenic mass for investigation. The pain was disturbing his daily activities as well as sleeping for associated with nausea and vomiting and constipation as well as loss of Appetit. However, there was no history of fever and other systemic review are unremarkable. 
By physical examination, the patient looks underweights but conscious, oriented with stable vital signs, not pale or jaundiced and his cardio-respiratory system was unremarkable. There was no lymphadenopathy or scares. There was large abdomen mass mildly-tender at the left hypochondrial area extend to left hypochondrium the umbilicus with rigid and smooth surface approximately $25 \mathrm{X} 18 \mathrm{~cm}$ in size extending from left upper quadrant of the abdomen till the hypochondrial region. By palpation, the mass was not mobile, hard consistency, round borders, and smooth surface. No lower limbs varicose vein, edam, or ischemic changes. All laboratory blood investigations revealed within normal range values for the blood count, liver and renal function tests, furthermore, all tumour markers were within normal ranges e.g. CA19-9 equal to 7.10 U/ml. Contrast CT (figure 1) scan showed normal liver and spleen size and density and revealed large heterogenous mass of pancreatic origin, from the body and tail measuring about $23 \mathrm{~cm}$ by $15 \mathrm{~cm}$ by $11 \mathrm{~cm}$. Displacing and compressing the stomach medially and spleen posteriorly and left kidney as well. Small bowel loops were also displaced to the Rt side. No lymph nodes were seen. Biopsy taken trans gastric under Endoscopic ultrasound guidance showed epithelial cells clusters a long with sheets of cells characterize by oval to round to spindly shaped nuclei in $\mathrm{Hg}$ background, however, no malignant cells demonstrated.

The had radical resection of the tumour thorough midline laparotomy. Extensive adhesion and infiltration to adjacent organ noticed encountered. the pancreatic mass was invading nearby organ but assessed to be resectable safely, therefore, radical en mass resection with extended left pancreatectomy, transverse colectomy. Splenectomy, adrenalectomy Patient had smooth recovery and no blood transfusion needed. Blood loss was estimated to be $450 \mathrm{ml}$; therefore, no blood transfused intraoperatively. Postoperatively, our patient progressed smoothly. The accurate gross size was $26 \times 17 \times 9 \mathrm{~cm}$ and the weight found to be $3.6 \mathrm{Kg}$ (figure 2).

The Histopathology demonstrated Intera-abdominal Fibromatosis by immunohistochemistry Positivity for B-catenin and C-Kit (CD117) and as important, negativity for S-100, Desmin, CD34, Bc12, CD99, ALK-1, Pan-Ck. All surgical resection margins were free of tumour no metastasis to adjacent organs and eleven lymph nodes examined from the specimen showed no malignant cell (figure 2).

Because the resection was curative and based on the histopathological nature of the tumour and the negative lymph node and no distant metastasis no chemo- or radiotherapy prescribed for the patient in tumour-board decision. The three-month follow-up CT scan and annual ultrasound for five years did not demonstrate any recurrence; therefore, curative management is achieved. Furthermore, the patient had excellent satisfaction and improvement of quality of life as he stated and confirmed by the oncologist too.

\section{Discussion:}

fibromatoses (Desmoid-type) tumour is rare benign fibrous tumours account approximately for $0.03 \%$ of all neoplasms in USA. Pancreas is even more rarely reported subset with approximately $5 \%$ of all desmoid-type fibromatoses [8-10].

The unique aggressive local invasion is characteristic of DT that render the tumour unresectacble or with high local recurrence, but not unknown to case distant metastases as thesis a benign tumour.

These tumours are more commonly present in young adolescent patient $[5,11]$. intraabdominally DT, occurs in around $8 \%$ of all DT and more likely to be in adults than paediatric age groups, affecting any part of the gastrointestinal and genitourinary tracts [12] the comments origin of intraabdominal DT are of mesenteric or retro peritoneum connective tissue. the pancreatic origin DT remain an extremely rare subset of DTs. The English literature from the 1980s report 27 pancreatic DT, therefor our case is the 28th case $[1,2,4,5$, $10,13-18]$.

clinical presentation of intraabdominal DTs is none specific in symptoms and signs. In general symptoms and signs are related anatomical location of the DT mass and/ or invasion of nearby structures causing bowel obstruction, ischemia, fistulas, gastrointestinal bleeding or perforation an ureteric obstruction. Pancreaticorigin DT behave like pancreatic cancer in presentation as asymptomatic mass or with nonspecific mild epigastric pain or painless jaundice if the tumor location is in the head or the uncinate process. [1,5]. Our 
patient presented with mild to moderated abdominal pain associated with nausea, weight loss and splenic mass for investigation. and possible complications [19].

Imagining is valuable especially CT scans and/ or MRI to determine invasiveness, aid in biopsy approach and to assess resectability but not diagnostic $[1,4,5]$.

In FNA histopathology as in our case the presence of spindle cells is common but not pathognomonic for DT. The Immunohistochemistry feature of intraabdominal DT characterize by a positive vimentin and $\beta$-catenin and as importantly negative for S100, CD34, and CD117 for the SMA [5]. Our case confirmed typical DT Immunohistochemistry criteria (Fig 3).

the immunohistochemistry is the mainstay of confirming DT with characteristic negative S100 protein, in most of the case with rare exceptions [20]. the presence of positive nuclear $\beta$-catenin staining is important diagnostic feature present in around $80 \%$ of the cases but not specific only for this tumour.

Unequally DT can be further confirmed by demonstrating mutations of $\beta$-catenin gene in exon 3 which is positive in $85 \%$ of the case $[11,13]$.

Favourable prognosis reported by Jia et al. with overall survival of $100 \%$ and disease-free survival of more than $80 \%$ [5]. however, accurate prognosis is not well reported and in sporadic cases reported high postoperative recurrence rate around 19-77\%, especially when associated with Gardner syndrome and FAP with recurrence rate up to $90 \%[5,16]$.

To date there we lack consensus or guidelines for management of DT. The first-line management of DT was and remain wide free margin complete resection (radical resection) given the aggressive nature and excessive local invasive behaviour of the tumour with the guidance of frozen section support considering the locally invasive behaviour of this tumour. Observation without surgical resection also called wait-and-see approach mainly in static tumour were recommended by some expert to avid morbidity and the possible high recurrence associated with surgery especially in asymptomatic cases with mild manageable symptoms.

As a possible second line of management and specifically in case of high-risk patients for surgical intervention or in advance stages an adjuvant systemic chemotherapy based mostly in combination of methotrexate, vinblastine, doxorubicin, and dacarbazine and /or radio- or molecular target therapy as Tamoxifen. The use of COX2 inhibitors e.g., NSAID and celecoxib demonstrated promising successes in some studies, however, the mechanism by which it achieves regression is not clearly reported $[4,5,16,19]$.

\section{Conclusion:}

DT in the pancreas is exceedingly rare form of already rare intraabdominal form desmoid type fibromatosis. by virtue of nonspecific clinical presentation or asymptomatic incident mass discovered DT diagnosis is considered challenging, secondly, the lack of specific clinical investigation. immunohistochemical staining is the main pathological diagnosis positive in $\beta$-catenin nuclear or negative as in S100 protein.

Radical-margin-free surgical resection remains the mainstay management approach while chemotherapy and/or radiotherapy are not effective and no standardized protocol of consensuses and considered the last resort for only high risk for or advance cases.

long-term follow up is warranted. Due to recurrence behaviour of the tumour.

According to midline English literature our case presents the largest pancreatic DT fibromatosis to be published.

Abbreviations:

Desmoid tumour (DT)

Computer tomography scan: CT scan

\section{Declarations:}


Ethics approval and consent to participate waived because it's a case reportConsent for publication: Written informed consent was obtained from the patient for publication of this case report and any accompanying images. A copy of the written consent is available for review by the Editor of this journal." Availability of data and material: Not applicable.

Financial disclosure: no conflict of interest, no financial funding

Competing interests: None of the authors has any conflict of interest to declare. or commercial associations for the author.

Funding: The study received no financial support.

Authors' contributions: Dr Hanan M Alghamdi: designed the study, collected data, analyzed data, wrote the paper.

Acknowledgement : thank you to Prof Elshawarabi histopathologist and Dr Ahmed Almerhabi endoscopist done EUS \& Biopsy.

\section{Reference:}

1. Gerleman R, Mortensen MB, Detlefsen S. Desmoid Tumor of the Pancreas: Case Report and Review of a Rare Entity. Int J Surg Pathol. 2015; 23(7):579-84.1

2. Gourgiotis S, Gemenetzis G, Villias C. Pancreatic Desmoid Tumour: Extremely Rare Presentation in an Elderly Patient. Hell J Surg. 2014; 86(6):378-81.

3. Leal RF, Silva PV, Ayrizono Mde L, Fagundes JJ, Amstalden EM, Coy CS. Desmoid Tumor in Patients with Familial Adenomatous Poliposis. Arq Gastroenterol. 2010; 47(4):373-8.

4. Jia C, Tian B, Dai C, Wang X, Bu X, Xu F. Idiopathic desmoid-type fibromatosis of the pancreatic head: case report and literature review. World J Surg Oncol. 2014;12:103.

5. Wang Y-C, Wong J-U. Complete remission of pancreatic head desmoid tumour treated by COX-2 inhibitor - a case report. World J Surg Oncol. 2016; 14:190.

6. Venkat D, Levine E, Wise WE. Abdominal pain and colonic obstruction from an intra-abdominal desmoid tumour. Gastroenterol Hepatol. 2010; 6: 662-5.

7. Joyce M, Mignanelli E, Church J. Ureteric Obstruction in Familial adenomatous polyposis-associated desmoid disease. Dis Colon Rectum. 2010; 53: 327-32.

8. Sturt NJ, Gallagher MC, Bassett P, Philp CR, Neale KF, Tomlinson IP, et al. Evidence for genetic predisposition to desmoid tumours in familial adenomatous polyposis independent of the germline APC mutation. Gut. 2004; 53:1832-6.

9. Penel N, Coindre JM, Bonvalot S, Italiano A, Neuville A, Le Cesne A, Terrier P, Ray-Coquard I, Ranchere-Vince D, Robin YM, Isambert N, Ferron G, Duffaud F, Bertucci F, Rios M, Stoeckle E, Le Pechoux C, Guillemet C, Courreges JB, Blay JY. Management of desmoid tumours: A Nationwide survey of labelled reference centre network in France. Eur J Cancer. 2016; 58: 90-6.

10. Slowik-Moczydlowska Z, Rogulski R, Piotrowska A, Maldyk J, Kluge P. Desmoid tumour of the pancreas: a case report. J Med Case Rep. 2015; 9: 104.

11. Kasper B, Ströbel P, Hohenberger P. Desmoid Tumors: Clinical Features and Treatment Options for Advanced Disease. Oncologist. 2011; 16: 682-93. www. TheOncologist.com.

12. Sakorafas GH, Nissotakis C, Peros G. Abdominal desmoid tumours. Surg Oncol. 2007; 16: 131-42.

13. Xu B, Zhu L-H, Wu J-G, Wang X-F, Matro E, Ni J-J. Pancreatic solid cystic desmoid tumour: case report and literature review. World J Gastroenterol. 2013;19(46):8793-8.

14. Zhang H, Yu S, Wang W, Cheng Y, Xiao Y, Lu Z, Chen J. Primary mesenchymal tumours of the pancreas in a single center over 15 years. Oncol Lett. 2016; 12: 4027-34.

15. Kim JY, Song JS, Park H, et al. Primary mesenchymal tumours of the pancreas: single-center experience over 16 years. Pancreas. 2014; 43: 959-68.

16. Hsueh C, Lin CY, Huang YC, Ho SY, Lee KW, Liu CK. Desmoid Mimicking Cystic Pancreatic Lesion: a case report. J Radiol Sci. 2014; 39: 91-5. 
17. Saida K, Miyazaki O, Matsuoka K, Watanabe T, Fujino A, Nosaka S. Pancreatic desmoid tumour in a 4-year-old male with hemihypertrophy. J Pediatr Surg Case Rep. 2015;3:244-347.

18. Tsukamoto Y, Imakita M, Nishitani A, Ito T, Izukura M. Hirota: Pancreatic desmoid-type fibromatosis with beta-catenin gene mutation-Report of a case and review of the literature. Pathol Res Pract. 2016; 212(5): 484-9.

19. Santos M, Rocha A, Martins V, Santos M. Desmoid Tumours in Familial Adenomatous Polyposis: Review of 17 Patients from a Portuguese Tertiary Center. J Clin Diagn Res. 2016; 10(10): PC01-5.

20. Ross JA, Zhang X. Desmoid-type fibromatosis. Atlas Genet Cytogenet Oncol Haematol. 2013; 17(8): $571-8$.

\section{Legend:}

Figure 1: A) a CT scan sagittal view with I/V \& oral contrast showing huge heterogenous hypodense mass originating from the pancreas, the body and the tail is replaced by the mass reported as: measuring $23 \mathrm{~cm}$ in craniocaudal, 15 in transverse plan and $11 \mathrm{~cm}$ in anteroposterior plan. B) Coronal section demonstrates the pancreatic mass compressing the left kidney. No evidence of intraabdominal or pelvic lymph node enlargement.

Figure 2: Intraoperative photo demonstrates the gross specimen including the distal pancreas, spleen, part of the transvers colon, and the adrenal gland. B). The cut surface is whirly homogenous tan to pink to white reaching spleen capsule but not invading it.

Figure 3: histopathology showing. A). Interaabdominal Fibromatosis

All surgical resection margins are free of tumor size $26 \times 17 \times 9 \mathrm{~cm}$.

B). Immunohistochemical staining of the tumor showing positive satin for anti-beta-catenin $\mathrm{x} 400$.

\section{Hosted file}

Figure 1 final.pdf available at https://authorea.com/users/409841/articles/519389-invasiveand-the-largest-pancreatic-tumour-of-rare-intraabdominal-desmoid-type-fibromatosis-withcurative-resection-case-report

\section{Hosted file}

figure 2 final.pdf available at https://authorea.com/users/409841/articles/519389-invasiveand-the-largest-pancreatic-tumour-of-rare-intraabdominal-desmoid-type-fibromatosis-withcurative-resection-case-report

\section{Hosted file}

figure 3 final.pdf available at https://authorea.com/users/409841/articles/519389-invasiveand-the-largest-pancreatic-tumour-of-rare-intraabdominal-desmoid-type-fibromatosis-withcurative-resection-case-report 